\title{
Internal solitons in the Andaman Sea: a new look at an old problem
}

\author{
J.C.B. da Silva ${ }^{\mathrm{ab} *} \&$ J.M. Magalhaes ${ }^{\mathrm{a}}$ \\ ${ }^{a}$ Faculdade de Ciências da Universidade do Porto - Departamento de Geociências, Ambiente e \\ Ordenamento do Território - Rua do Campo Alegre 687, 4169-007 - Porto, Portugal; \\ ${ }^{\mathrm{b}} \mathrm{CIMAR} / \mathrm{CIIMAR}$ - Interdisciplinary Centre of Marine and Environmental Research, University of \\ Porto, Rua dos Bragas 289, 4050-123 Porto, Portugal
}

\begin{abstract}
When Osborne and Burch ${ }^{[1]}$ reported their observations of large-amplitude, long internal waves in the Andaman Sea that conform with theoretical results from the physics of nonlinear waves, a new research field on ocean waves was immediately set out. They described their findings in the frame of shallow-water solitary waves governed by the K-dV equation, which occur because of a balance between nonlinear cohesive and linear dispersive forces in a fluid. It was concluded that the internal waves in the Andaman Sea were solitons and that they evolved either from an initial waveform (over approximately constant water depth) or by a fission process (over variable water depth). Since then, there has been a great deal of progress in our understanding of Internal Solitary Waves (ISWs), or solitons in the ocean, particularly making use of satellite Synthetic Aperture Radar (SAR) systems. While two layer models such as those used by Osborne and Burch ${ }^{[1]}$ allow for propagation of fundamental mode (i.e. mode-1) ISWs, continuous stratification permits the existence of higher mode internal waves. It happens that the Andaman Sea stratification is characterized by two (or more) maxima in the vertical profile of the buoyancy frequency $\mathrm{N}(\mathrm{z})$, i.e. a double pycnocline, hence prone to the existence of mode-2 (or higher) internal waves. In this paper we report solitary-like internal waves with mode-2 vertical structure co-existing with the large well know mode-1 solitons. The mode- 2 waves are identified in satellite SAR images (e.g. TerraSAR-X, Envisat, etc.) because of their distinct surface signature. While the SAR image intensity of mode-1 waves is characterized by bright, enhanced backscatter preceding dark reduced backscatter along the nonlinear internal wave propagation direction (in agreement with Alpers, $1985^{[2]}$ ), for mode-2 solitary wave structures, the polarity of the SAR signature is reversed and thus a dark reduced backscatter crest precedes a bright, enhanced backscatter feature in the propagation direction of the wave. The polarity of these mode- 2 signatures changes because the location of the surface convergent and divergent zones is reversed in relation to mode-1 ISWs. Mode-2 ISWs are identified in many locations of the Andaman Sea, but here we focus on ISWs along the Ten Degree Channel which occur along-side large mode-1 ISWs. We discuss possible generation locations and mechanisms for both mode-1 and mode-2 ISWs along this stretch of the Andaman Sea, recurring to modeling of the ray pathways of internal tidal energy propagation, and the P. G. Baines ${ }^{[3]}$ barotropic body force, which drives the generation of internal tides near the shallow water areas between the Andaman and Nicobar Islands. We consider three possible explanations for mode-2 solitary wave generation in the Andaman Sea: (1) impingement of an internal tidal beam on the pycnocline, itself emanating from critical bathymetry; (2) nonlinear disintegration of internal tide modes; (3) the lee wave forming mechanism to the west of a ridge during westward tidal flow out of the Andaman Sea (as originally proposed by Osborne and Burch for mode-1 ISWs). SAR evidence is of critical importance for examining those generation mechanisms.
\end{abstract}

Keywords: Internal Waves, Synthetic Aperture Radar, Andaman

*jdasilva@fc.up.pt; phone: +351 220402476; fax: +351 220402490

Remote Sensing of the Ocean, Sea Ice, Coastal Waters, and Large Water Regions 2016, edited by Charles R. Bostater, Jr., Xavier Neyt, Caroline Nichol, Oscar Aldred, Proc. of SPIE Vol. 9999, $999907 \cdot$ (C) 2016 SPIE · CCC code: 0277-786X/16/\$18 · doi: 10.1117/12.2241198 


\section{INTRODUCTION}

Extraordinarily large internal waves are characteristic of the Andaman Sea of the Indian Ocean. They had been observed even before the first scientific accounts of internal waves had been reported. Seafarers passing through the Strait of Malacca on their journeys between India and the Far East have often noticed in the Andaman Sea between the Nicobar Islands and the north east coast of Sumatra bands of choppy waters or ripplings reaching from horizon to horizon. An early description of these surface manifestations of internal waves is provided in the book of Mauray which was published in 1861 and which is quoted in Osborne and Burch (1980) ${ }^{[1]}$ : "The ripplings are seen in calm weather approaching from a distance, and in the night their noise is heard a considerable time before they come near. They beat against the sides of a ship with great violence, and pass on, the spray sometimes coming on deck; and a small boat could not always resist the turbulence of these remarkable ripplings". It is now understood that these descriptions correspond to surface manifestations of wind waves modulated by the presence underneath of large amplitude internal waves, or trains of solitons. While these waves propagate in the interior of the ocean as disturbances of density, to first order, they are not associated with an elevation or depression of the sea surface, alike the surface waves. Their surface manifestation is noticed by variations of the sea surface roughness, which in the Andaman Sea takes the form of meter-scale waves with considerable amplitude and wave breaking. This is a consequence of the fast propagation of these large internal waves in deep water and of the strong stratification in the equatorial Andaman Sea, regulating a resonant coupling between internal waves and meter-scale waves.

Perry and Schimke ${ }^{[4]}$ were the first to conduct oceanographic measurements of these waves from a ship, confirming that these bands of choppy water in the Andaman Sea were associated with solitons, or Internal Solitary Waves (ISWs). Later, Osborne and Burch ${ }^{[1]}$ analyzed oceanographic data collected by the Exxon Production Research Company in the southern Andaman Sea with the aim to assess the impact of underwater currents associated with oceanic internal waves on drilling operations carried out from a drill ship. Their oceanographic measurements showed that the waves appear in packets, or wave trains, and that the time interval between the first waves in a wave packet was typically 40 minutes and then decreased towards the end. The internal wave amplitudes (peak-to-trough vertical distance) also decreased towards the end of each packet, and was estimated to be around $60 \mathrm{~m}$, i.e., warm water in the upper water layer was pushed down by the internal wave by $60 \mathrm{~m}$. The bands of surface breaking waves (1.8 meter high) associated with each internal wave in a given packet extended from horizon to horizon and were 600 to $1200 \mathrm{~m}$ wide, being detected from space on optical images $^{[5]}$. It was also recognized by that time that the internal waves have tidal origin and their average phase speed

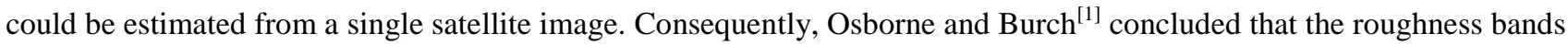
observed visually and from space are caused by ISWs which can be described by the soliton theory developed by Korteweg - de Vries ${ }^{[6]}$.

Propagation of internal waves (IWs) is naturally bounded, both above and below by the ocean's surface and bottomthus confining them within a natural waveguide. However, the ocean's pycnocline can also be seen as another form of waveguide, since it confines the higher frequency oscillations into interfacial waves (e.g. the ISWs seen in satellite imagery). The vertical confinement imposed by these boundaries results in horizontally propagating waves, which nonetheless have a standing character in the vertical. In this case, a classical Sturm-Liouville problem arises if the linear equations of motions (under the Boussinesq and traditional approximations) are solved with boundary conditions for zero vertical velocities at the surface and bottom of the ocean ${ }^{[7]}$. Therefore, IW solutions with an infinite number of modes can either exist in nature on their own or in any number of combinations (see e.g. Gerkema and Zimmerman ${ }^{[8]}$ for a detailed description on IW dynamics). The first case, usually results in low mode propagation of IWs (like interfacial IWs of tidal frequency, i.e. Internal Tides - ITs), while the second may result in an increasing superposition of modes, which makes the spatial appearance of the IW field to resemble a beam structure (with energy that propagates along beams or rays). In the particular case of SAR imagery, ISWs are generally described as interfacial mode 1 waves of depression (propagating along the pycnocline, and depressing it) that are usually observed as wave packets of ISWs in the Andaman Sea. However, recent attention has been given to a different set of observations, where SAR surface 
signatures are not consistent with mode 1 ISWs, but rather suggest mode 2 solitary-like waves (for simplicity referred to in this paper as mode 2 ISWs). The traditional view is that first-mode IWs are most commonly observed in the ocean, but higher-mode waveforms also occur under the proper conditions. A mode-2 wave is best thought of in terms of a threelayer stratified ocean, when the middle-layer thickness is relatively thinner than the upper and lower layers. In this configuration, the wave travels as a bulge in the middle layer, displacing isopycnals upward into the upper layer and downward into the bottom layer (see e.g. [9]).

Sea surface signatures of mode 1 and mode 2 ISWs can be unambiguously identified in SAR images provided the waves propagate in relatively deep water (when the lower layer is significantly deeper than the mixed layer depth). If interfacial waves (in a two layer system) travelling along the pycnocline are waves of depression (which is often the case in deep waters), then mode 1 ISWs will be revealed in SAR images by bright bands preceding darker ones in their direction of travel (see Alpers ${ }^{[2]}$ ). However, mode 2 ISWs will have precisely the opposite contrast in the SAR, since their radar signature consists of dark bands preceding bright bands in their direction of propagation (see Fig. 1).

This is because surface velocity fields induced by travelling ISWs of different modes can create different convergence and divergence patterns, which then modulate the surface roughness and thus the intensity of the radar backscatter signal. In particular, a mode 2 ISW travelling along the thermocline will generate a divergence pattern at the surface, followed by a convergence pattern in the propagation direction (see Fig. 1). It is this dynamical feature (reversed with respect to mode 1 ISWs) that produces the characteristic dark bands ahead of the bright bands in their direction of travel.

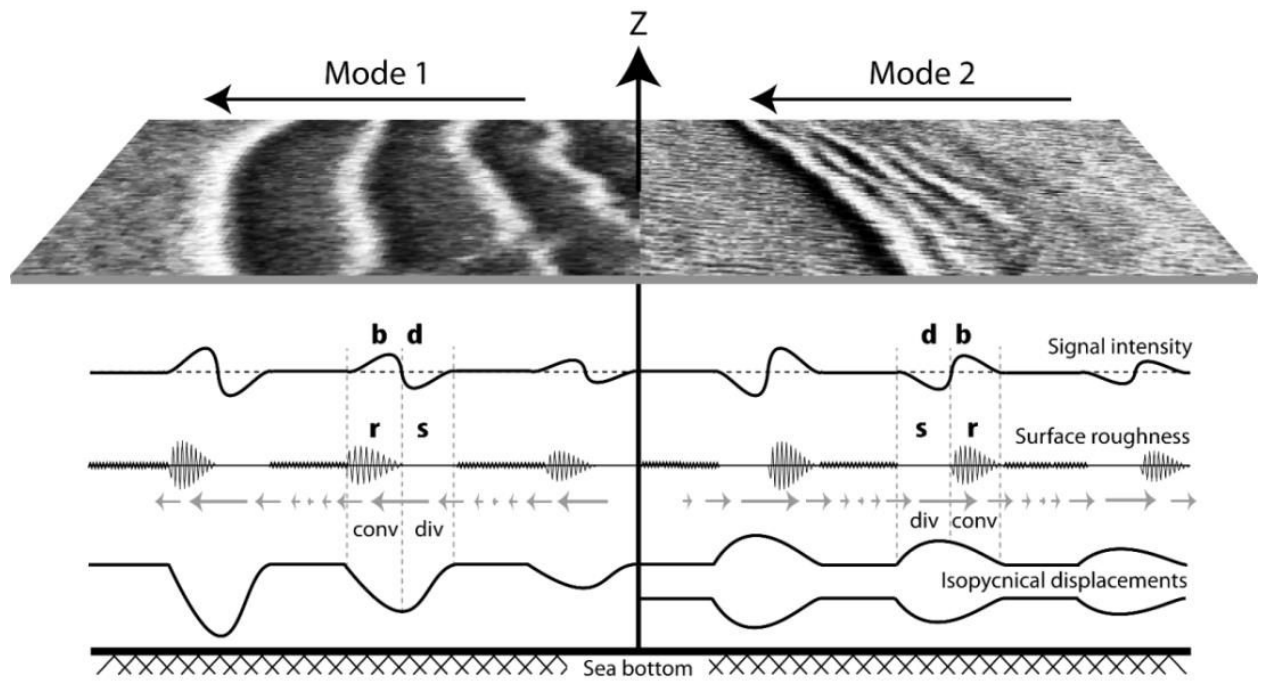

Fig. 1. Examples of SAR images with sea surface signatures of mode 1 and 2 ISWs are shown at the top (left and right, respectively). The ISWs are assumed to be moving from right to left. From top to bottom the horizontal profiles represent the following features: SAR intensity profile along the ISWs, with bright enhanced backscatter (b) preceding dark reduced backscatter (d) in the direction of propagation for mode 1 waves; surface roughness representation indicating how rough (r) and smooth (s) the surface is along an ISW wave packet; surface current variability induced by ISWs (note indication of convergence and divergence fields near the surface); isopycnical displacements produced by ISW propagation. Note how the SAR signatures, surface roughness, current fields and convergence and divergence patterns are all reversed between mode 1 (on the left) and mode 2 (on the right) ISWs.

Research studies on mode 2 ISWs generated by IW rays hitting a pycnocline have been recently presented using numerical models and laboratory experiments (see [10] and [11], respectively). These studies were focused on the local generation mechanism (see Sect. on Methodology), and have also addressed the generation of higher mode waves by IW rays. Grisouard et al. ${ }^{[10]}$ used a (nonlinear and non-hydrostatic) numerical model and concluded that higher mode ISWs (particularly mode 2) may also be generated by an IW beam hitting a pycnocline at an angle. Da Silva et al. ${ }^{[12]}$ found 
mode 2 ISW generation in the Mascarene Ridge of the Indian Ocean consistent with the hypothesis in [10]. In their studies, high mode waves are favored by a strongly stratified pycnocline or, equivalently, by short-wavelength IT beams. The former is certainly the case in the Andaman Sea. Laboratory measurements of ISWs generated by an internal wave beam were presented in [11], and are in agreement with the results of [10]. Following these numerical and laboratory studies, we will show in this paper satellite observations of mode 2 ISWs, which are consistent with local generation by an internal tide (IT) beam impinging on the ocean pycnocline from above after reflection from the sea surface.

\section{METHODOLOGY}

Internal tidal energy generated at ocean ridges or the shelf-break may radiate away either horizontally in the form of interfacial ITs that propagate along the thermocline, or as internal tidal beams that propagate into the deep stratified ocean below. Therefore, large interfacial ITs may form in the thermocline directly above bottom topography and evolve (through nonlinear processes) to higher-frequency ISW packets ${ }^{[13]}$. Another possibility is that the IT energy may propagate obliquely with a slope to the horizontal, giving rise to a second generation mechanism known as "local generation" (see e.g. [10] and [11]; and referenced therein). According to this generation mechanism, a beam (or ray) of IT energy may be generated at "critical" slope regions of ocean ridges where the bottom topographic slope matches the slope of the IT ray paths, and propagates at an angle $(\theta)$ to the horizontal along a vertical plan of the ocean interior (see Eq. 1) where $\sigma$ is the tidal frequency, $f$ the Coriolis parameter and $N$ the buoyancy frequency).

$$
\tan (\theta)=\left(\frac{\sigma^{2}-f^{2}}{N^{2}-\sigma^{2}}\right)^{1 / 2}
$$

These rays reflect from the seafloor ${ }^{[14],[15]}$ and the sea surface ${ }^{[12]}$, and interact with the thermocline from below or above, respectively, causing large oscillations there, and "locally" generating ISWs (far from where the beam is initially generated). This hypothesis was originally proposed to explain some ISW observations in the northern Bay of Biscay, where it was studied for the first time, and where SAR imagery helped to clearly distinguish a bimodal distribution of ISW $^{[16]}$. Several other studies have emerged since, which have also identified locally generated waves in other regions of the world's oceans. Namely, the southern Bay of Biscay ${ }^{[17]}$, the Estremadura Promontory ${ }^{[18]}$, the Mozambique Channel ${ }^{[19]}$, and more recently the Mascarene Ridge of the Indian Ocean ${ }^{[12]}$, have also been found as major hotspot regions for the local generation mechanism. In this paper we will show clear evidence (based on remote sensing SAR images and modelling results) that observations of mode 2 ISWs along the Ten Degree Channel of the Andaman Sea are consistent with local generation, to the east of a double ridge between the Andaman and Nicobar Islands.

To examine the generation issue and assert the exact locations where ISWs are generated, the methodology that is usually presented in the literature consists of two main steps. On the one hand, the Baines ${ }^{[3]}$ barotropic tidal forcing is often used to search and identify hotspot regions of ISWs within a given study area. It has proved to be a valuable indicator in several other independent studies, particularly to study the possibility of the local generation mechanism. For instance [17] and [18] have used this method to investigate the local generation mechanism in the southern Bay of Biscay (near Cape Finisterre), and in the southern slopes of the Estremadura Promontory (off the west Iberian shelf). In addition, there have been other authors that also used this technique over the last decades, as a valuable indicator of where large ITs may be generated ${ }^{[20] ; ~[21] ; ~[22] . ~ O n ~ t h e ~ o t h e r ~ h a n d, ~ r a y-t r a c i n g ~ t e c h n i q u e s ~ a r e ~ u s e d ~ t o ~ s e e ~ i f ~ t h e ~ l o c a t i o n s ~}$ where ISWs first appear are consistent with the local generation mechanism (i.e. if the first ISW packets appear a few kilometers ahead of the expected impact of an IT beam with the pycnocline). Note that, ITs in a continuously stratified ocean can be described by beams or rays that follow characteristic pathways (along which their energy can propagate see Eq. 1). In other words, this means that when the bottom slopes match the local value of $\tan (\theta)$ (called a critical region) the generation of ITs is more pronounced, particularly if the barotropic currents are strong. In such cases, the 
direction of the forcing barotropic flow is then coincident with the motion plane for free internal waves, resulting in resonant conditions and enhanced generation of the ITs and ISWs.

The barotropic forcing term (following [3]) for ITs, resulting from the interaction of bottom topography and tidal flow, can be defined as

$$
F=z \cdot N^{2}(z) \int Q \cdot d t \nabla\left(\frac{1}{h}\right)
$$

where $\mathrm{z}$ is the vertical coordinate (positive upwards), $\mathrm{Q}$ is the barotropic mass flux vector $\mathrm{Q}=(\mathrm{uh}, \mathrm{vh})$ with $\mathrm{u}$ and $\mathrm{v}$ being the zonal and meridional components of the barotropic velocity, and $\mathrm{h}$ is the ocean depth. This means that $\mathrm{F}$ can be analytically integrated provided that $\mathrm{Q}$ is previously known, and for that purpose the components of the barotropic velocity vector were taken from the $1 / 12^{\circ}$ resolution OTIS model (Oregon state university Tidal Inversion Software, developed in [23]). The model included 11 tidal constituents $\left(\mathrm{M}_{2}, \mathrm{~S}_{2}, \mathrm{~N}_{2}, \mathrm{~K}_{2}, \mathrm{~K}_{1}, \mathrm{O}_{1}, \mathrm{P}_{1}, \mathrm{Q}_{1}, \mathrm{M}_{4}, \mathrm{MS}_{4}, \mathrm{MN}_{4}\right.$ ), of which the $\mathrm{M}_{2}$ and $\mathrm{S}_{2}$ tidal constituents (periods of 12.42 and $12.00 \mathrm{~h}$, respectively) are the most important of the barotropic tide in this study region. The bathymetry data is part of the one minute global bathymetry from Smith and Sandwell ${ }^{[24]}$, and $\mathrm{N}(\mathrm{z})$ is assumed to be spatially constant in the horizontal.

\section{RESULTS}

The case study addressed in this paper reports mode-2 short-period internal waves propagating along the Ten Degree Channel of the Andaman Sea, which were identified in an ENVISAT ASAR image dated 2 January, 2007 (presented in Fig. 3). The area covered by this image is depicted in the map shown in Fig. 2a (in light blue shade) along with the bathymetry of the region and the locations of Conductivity Temperature Depth (CTD) stations used to assess the vertical stratification (Brunt-Väisälä frequency presented in Fig. 4b). The bottom topography along the Ten Degree Channel cross section is characterized by a double ridge system relatively shallow compared to other bottom features of the Andaman Sea whose depth is some $800 \mathrm{~m}$ or less.

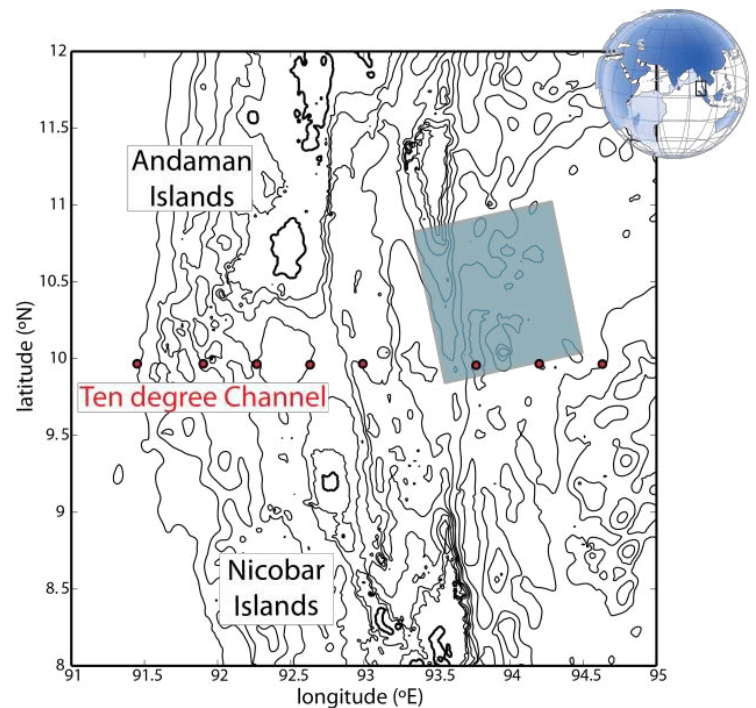

Fig. 2a. Map of the study region (Andaman Sea). Red dots represent locations of CTD stations used to calculate the average vertical stratification (see text for details). Light blue rectangle shows the area of the SAR image shown in Figure 3. Isobaths of 200, 500, 1000, 1500, 2000, 3000 and $4000 \mathrm{~m}$ depth are shown.

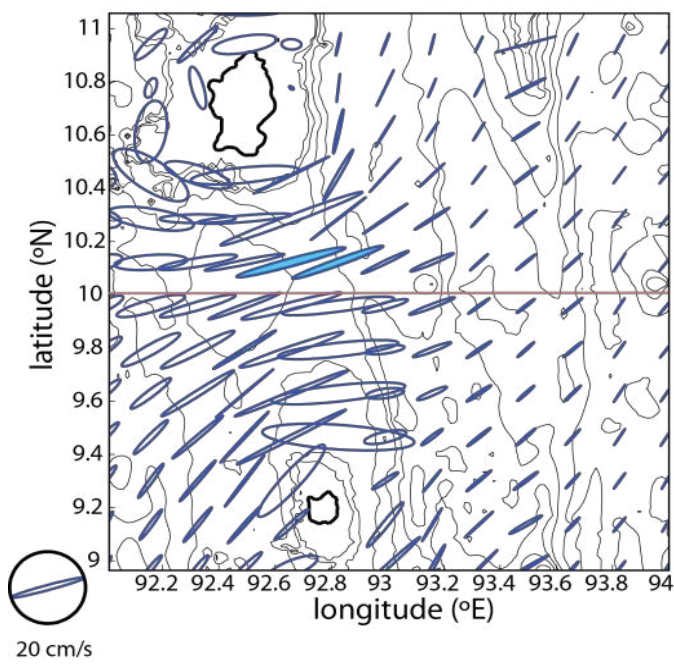

Fig. 2b. Tidal ellipses calculated from OTIS (see text for details) showing tidal flow across a double ridge at the Ten degree Channel. Note the scale of the tidal currents on the lower left corner. Same isobaths as in Fig. 2a are shown. Two tidal ellipses are filled in light blue over the Eastern Ridge, where generation of ITs is expected. 
In Fig. 2b a map showing tidal ellipses for the semi-diurnal tidal cycle closest in time to the ASAR image acquisition is presented, showing that the tidal ellipses become preferentially aligned in the across-slope direction of the double ridge that exists at the Ten Degree Channel in between longitudes 92.0 and $92.5^{\circ} \mathrm{E}$. This preferential across-slope tidal forcing is important as it indicates the across-ridge direction as a possible candidate for internal wave generation.

Figure 3 shows the Envisat ASAR image dated 02.01.2007 acquired at 15:48 UTC in Precision Image mode (spatial resolution approximately 20 meters). Detailed inspection of the SAR signatures in the same fashion as [11] reveals that the ISW packets shown (having crests approximately parallel to the double ridge system) propagate into the Andaman Sea to the East of the ridges and are consistent with mode-2 ISW-like waves. The earliest evidence of mode-2 SAR signatures present in this image appears to the East of the Eastern Ridge, whose crests are aligned with the Ridge but some $40-50 \mathrm{~km}$ to the East. The Eastern Ridge is also characterized by the strongest tidal currents in the study area, with values of approximately $20 \mathrm{~cm} / \mathrm{s}$ (see Fig. 2b). Figs. $3 \mathrm{~b}$ and $3 \mathrm{c}$ show details of the radar backscatter modulations along internal wave propagation, revealing that dark bands precede bright bands in their direction of propagation. Considering the ocean depths there, this indicates that the internal waves are of the second mode, since they cannot be mode-1 ISWs of depression. In many other SAR images of the same kind we found that mode-2 ISWs are characteristic of this stretch of the Andaman Sea (i.e. along the Ten Degree Channel East of the Eastern Ridge).

To begin to explain these ISW patterns in more detail, we now turn to theoretical considerations. Internal tides in a continuously stratified ocean can be described by beams or rays that follow characteristic pathways (along which their energy can propagate) with a slope to the horizontal given by Eq. 1. When the seafloor slope matches the local value of $\tan (\theta)$ (a critical region) generation of ITs is pronounced, particularly if the barotropic currents are strong. In such cases, the direction of the forcing barotropic flow is then coincident with the motion plane for free internal waves, resulting in resonant conditions and enhanced generation of the ITs. Body force calculations shown in Fig. 4a, based on local stratification measurements shown in Fig. 4b, confirm the Eastern Ridge as a likely candidate to explain internal wave generation, since the body force is elevated there (see [3]).

Figure $4 b$ shows an average profile of the Brunt-Väisälä frequency for different locations along the Ten Degree Channel. The profile represents an average stratification for October 1995 based on 8 CTD stations along the Ten Degree Channel (locations of CTD stations shown in Figure 2a, red dots). The IT ray paths calculated according to this average stratification are presented in Figure 5 and illustrate a possible generation mechanism for these mode-2 internal wave trains. IT rays may form near critical bathymetry, i.e. bathymetry whose slope is nearly the same as the IT ray characteristics, and energy propagates upwards from the Eastern Ridge. The energy eventually impinges the pycnocline from above (after reflecting from the sea surface) and is thought to give origin to the mode- 2 wave trains visible in the SAR image in Figure 3. 


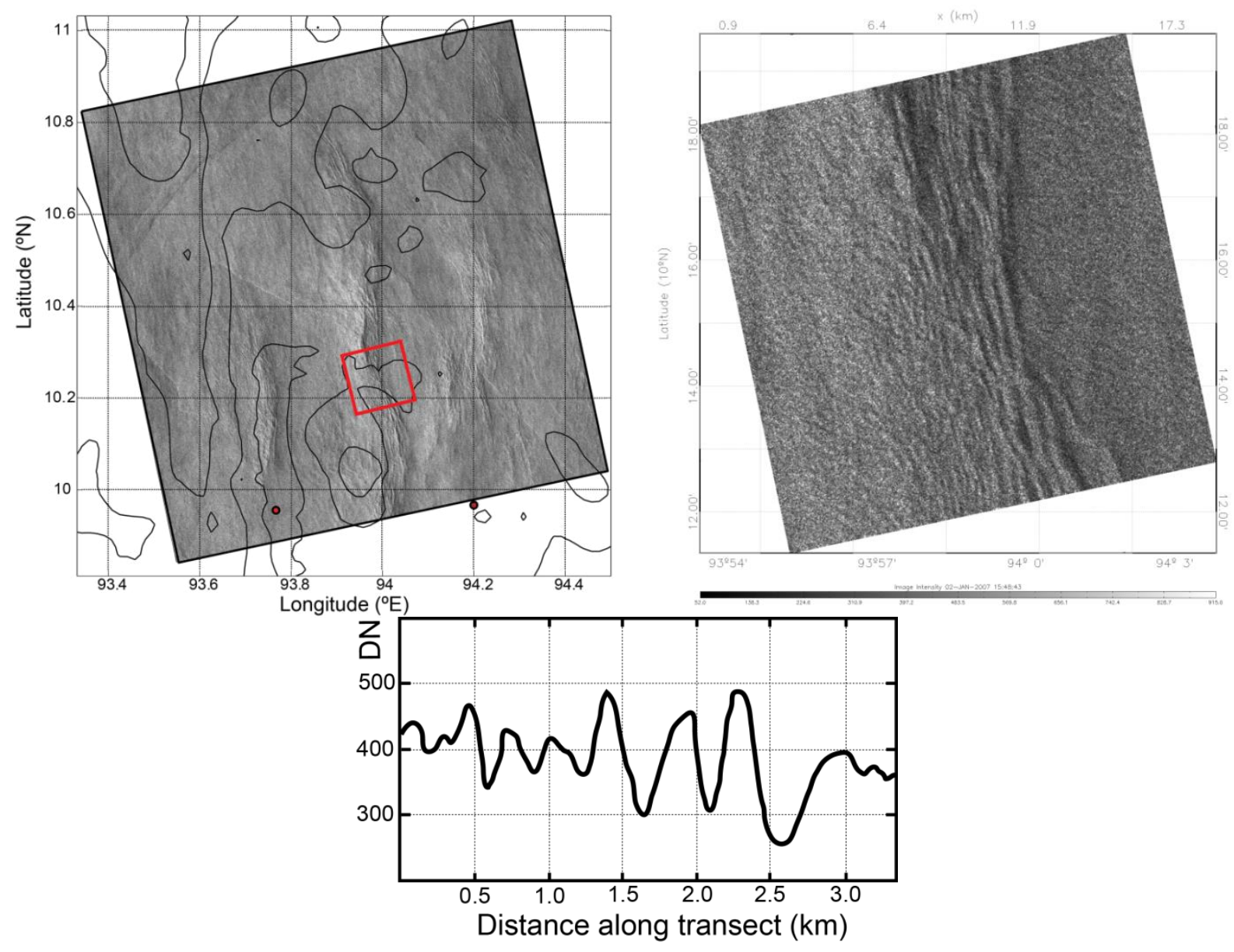

Fig. 3. Top left (a) Envisat ASAR IMP dated 02.01.2007 whose dimensions are approximately $100 \mathrm{x} 100 \mathrm{~km}$. Isobaths are $1000,2000,3000$ and $4000 \mathrm{~m}$. Red dots are locations of CTD stations used to calculate average stratification (see text for details). Red rectangle indicates zoom area shown in top right, part (b) SAR signature of a train of mode-2 ISWs. Bottom (c) Representative image transect showing radar backscatter cross-section anywhere across the mode- 2 wave train. Note how the first wave in the train is characterized by a negative backscatter variation first, followed by a positive one. 

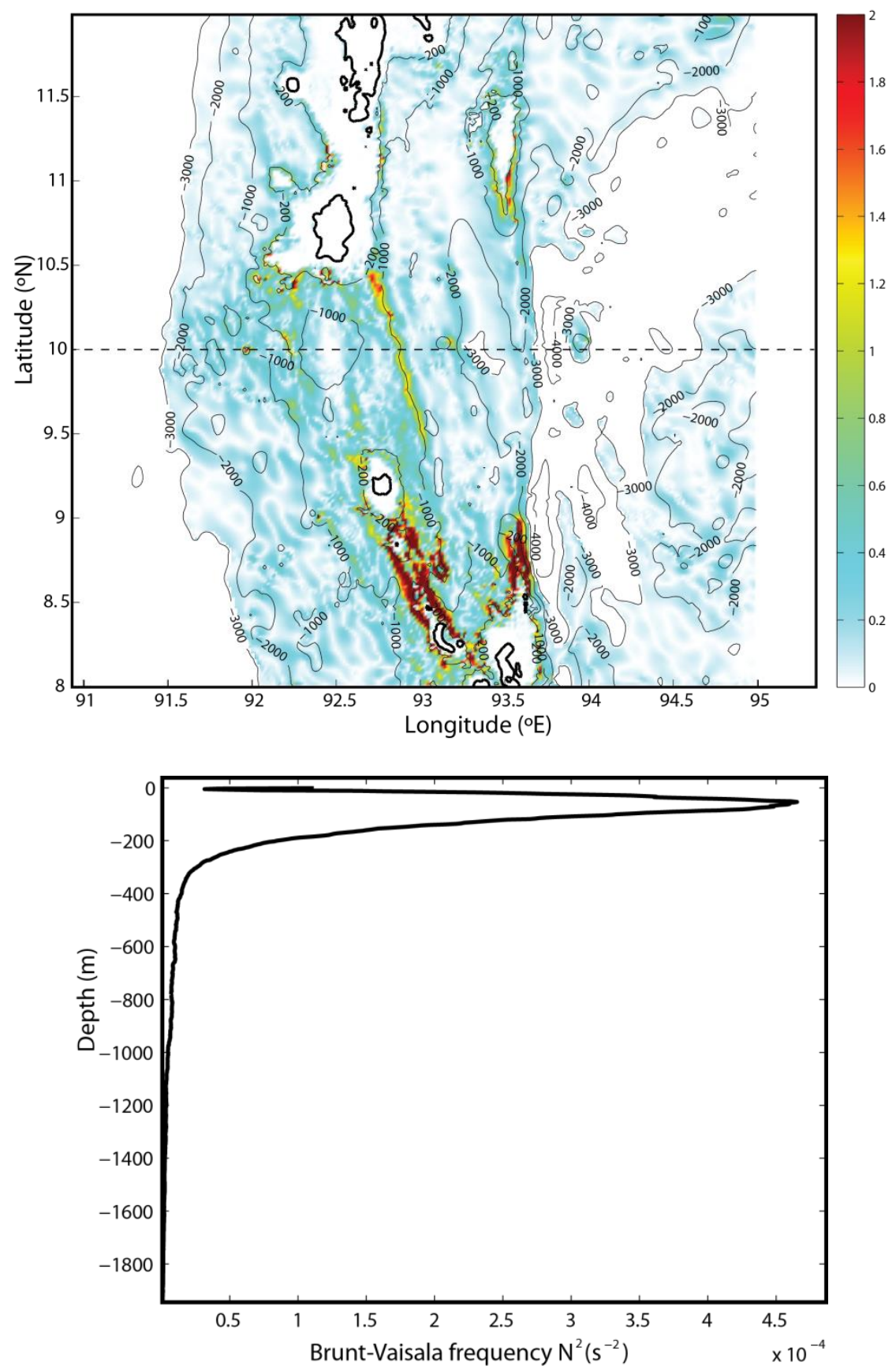

Fig. 4. Top: map showing the maximum depth integrated body force of Baines ${ }^{[3]}$ achieved during a complete semidiurnal tidal cycle (see text for details). Colors represent the strength of the body force (see color code on the right hand side of the map) and units are in $\mathrm{m}^{2} \cdot \mathrm{s}^{-2}$. Bottom: averaged vertical profile of stratification along the Ten degree Channel of the Andaman Sea used to compute the body forcing and the ray tracing of IT rays. 


\section{DISCUSSION}

We now discuss in more detail the SAR signatures of the ISWs and make suggestions as to possible generation mechanisms. Figure 3a shows a full frame of an Envisat ASAR image from 2 January 2007 (marked in light blue in Figure 2a) corresponding to an area of approximately $100 \times 100 \mathrm{~km}$. Several individual wave packets separated by distances of approximately $25-30 \mathrm{~km}$ from each other along their propagation direction can be identified in the radar image, suggesting internal tides with crests that are parallel and horizontal (Sverdrup waves), and whose wavelengths are long compared with the depth of the ocean.
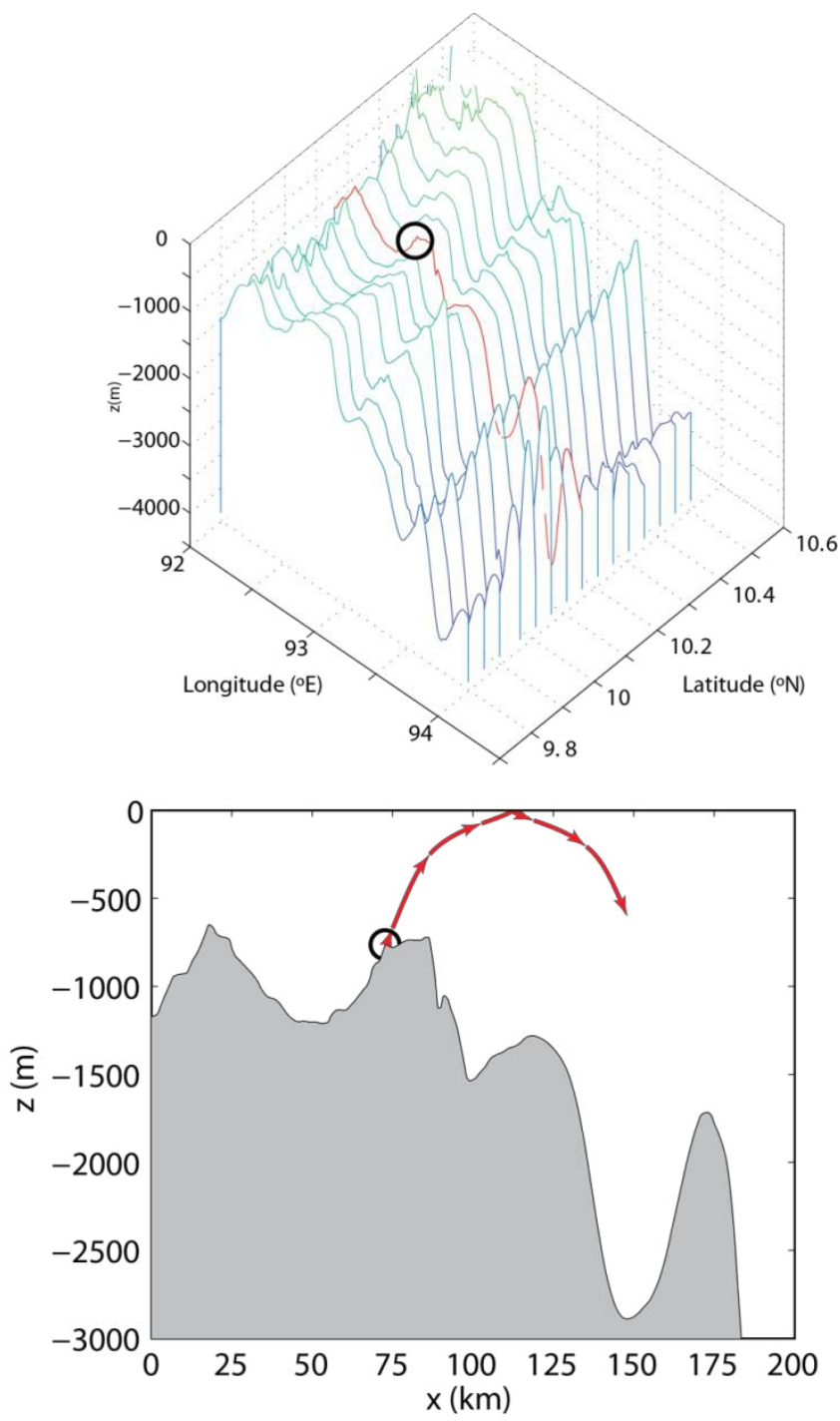

Fig.5. Ray tracing diagram showing a possible pathway of internal tide energy along a particular cross section of the Ten Degree Channel in the Andaman Sea. Top panel: 3D view of the system of ridges along the Ten Degree Channel of the Andaman Sea (the dark circle depicts location of critical topography from which rays emanate upward, as in part b). The red profile in part a) corresponds to the bathymetry cross section used in part b) to ray trace simulation. Bottom panel: Internal Tide of $\mathrm{M}_{2}$ frequency ray path calculated according to Eq. 1 and the vertical stratification profile in Fig. 4b.

The packets of short-period ISWs are interpreted as propagating from West to East along with the internal tide, i.e. in a direction (070 - $090^{\circ}$ clockwise from North) normal to the double ridge system. Following usual procedure in [19] and 
assuming that they are generated at consecutive cycles of the semi-diurnal $\mathrm{M}_{2}$ tide, their average phase propagation speed is $0.56 \mathrm{~m} / \mathrm{s}-0.67 \mathrm{~m} / \mathrm{s}$. The radar signatures of the ISWs (see Fig. $3 \mathrm{~b}$ and Fig. 3c) are suggestive of mode-2, or higher mode IWs, while their rank-order wavelength distribution indicates a nonlinear character as in [1].

Similar observations in other regions of the ocean (e.g. Bay of Biscay ${ }^{[25]}$ ), where the interfacial internal tide (period $12.42 \mathrm{~h}$ ) is often characterized by wave structures of higher frequency (periods of approximately $20 \mathrm{~min}$ and wavelengths of $1 \mathrm{~km}$ ) which appear mainly in the troughs of the internal tide, lead to the hypothesis that the shorterperiod IWs may be generated close to the bottom forcing. The assumption often has been that "the ISWs are generated because the leading slope of the internal tidal trough initially steepens due to nonlinearity, as it propagates from the shelf-break or ridge upper slopes into the deep ocean" [25] [26]. The more slowly propagating shorter waves may then be partially trapped in the internal tidal trough, where a favorable current structure effectively advects them along (i.e. both short and large wave structures may be coupled, moving with the same phase speed). The sea-surface signatures of the higher-wavenumber structures can be observed in the radar, while the long internal tide is usually not observed. Given this interconnectivity between internal tide and short-period ISWs, we next discuss alternative generation mechanisms for both these type of waves in the Andaman Sea.

An interfacial internal tide is usually generated by tidal flow over steep bottom slopes and results from upstream blocking $^{[27]}$, or some sort of simple lee wave mechanism ${ }^{[25]}$. It is not an easy task to confidently propose a generation mechanism for the internal tide without in situ measurements, model results or analysis of a large number of satellite images of the study region. However, a few things can be said from scrutiny of the single SAR image presented in Fig. 3 and assessment of typical stratification in the Andaman Sea (see Fig. 4b). Linear theory can be used to investigate the phase speeds and wavelengths of the natural vertical modes that are allowed in the system. Continuous stratification must be considered, to take into account the full density structure, as the density below the thermocline increases slowly with increasing depth (see Fig. 4b). Solving a standard Boundary Value Problem (BVP) of the Taylor-Goldstein equation (3) with appropriate boundary conditions, provides the phase speeds, $c$, and wavelengths, $\lambda$, of the first few vertical modes of the semi-diurnal internal tide (see results in Table 1). For this a flat bottom at depth $1800 \mathrm{~m}$ is assumed (see Fig. 3a).

Table 1. Phase speeds, c, and wavelengths, $\lambda$, of the first four vertical modes of the semi-diurnal internal tide.

\begin{tabular}{|c|c|c|c|c|}
\hline $\begin{array}{c}\text { Mode } \\
\text { number }\end{array}$ & $\mathbf{1}$ & $\mathbf{2}$ & $\mathbf{3}$ & $\mathbf{4}$ \\
\hline $\mathrm{c}(\mathrm{m} / \mathrm{s})$ & 2.37 & 1.26 & 0.78 & 0.59 \\
\hline$\lambda(\mathrm{km})$ & 106 & 56 & 35 & 26 \\
\hline
\end{tabular}

As seen above, the average phase speed retrieved from the image is $0.56 \mathrm{~m} / \mathrm{s}-0.67 \mathrm{~m} / \mathrm{s}$, assuming the same frequency of the $\mathrm{M}_{2}$ internal tide. This means that the fourth mode gives the most realistic linear phase speed for the horizontally propagating internal tides observed in the SAR image. It also means that the full density structure results in a generation and conversion of internal tidal energy into higher internal modes and allows rays to penetrate the ocean at an angle given by the internal wave characteristic ${ }^{[3]}$. Consequently, we evaluate the possibility of the local generation of the shorter-period ISWs of the second mode by beam scattering into the pycnocline in the same fashion as proposed in [10]. The ray tracing simulation presented in Fig. $5 \mathrm{~b}$ is geometrically consistent with this hypothesis, since the IT rays propagate upward and eastward from the summit of the Eastern Ridge, intersecting the pycnocline for the first time some $25 \mathrm{~km}$ upstream, where the energy first is transmitted across the pycnocline in the upward direction, reflects from the sea surface further upstream and then travels downward intersecting the pycnocline again some $50 \mathrm{~km}$ upstream from the critical slopes where the IT ray originates. It is suggested that, in this second interaction with the pycnocline, mode-2 ISWs are generated in a similar way as suggested by [32], [10] and [12]. Further research is needed to confirm or dismiss this hypothesis, for instance recurring to high resolution fully nonlinear and nonhydrostatic numerical modelling (e.g. 
with the MITgcm). Other generation mechanisms such as the lee wave mechanism ${ }^{[33]}$ or nonlinear evolution of interfacial internal tides ${ }^{[34]}$ should also be investigated with scrutiny. Note that these mode-2 ISWs are long-lived features in this stretch of the Andaman Sea (and live for at least $24 \mathrm{~h}$ ), since three consecutive wave packets are observed in this particular image and other images of the same kind. This raises an interesting question: could these short-period mode-2 ISWs be coupled with the internal tide, as their phase speeds are close to each other? To investigate this hypothesis we next show that the linear phase speed corresponding to the mode-2 short-period ISWs is indeed close to the much larger-scale mode-4 internal tidal wave.

Similar coupling between internal tides and short-period ISW packets were reported in the Bay of Biscay, where mode-1 ISWs are generated locally and coexist with interfacial internal tides consistent with the third or fourth vertical modes ${ }^{[16]}$ ${ }^{[25]}$. Again, a standard BVP is solved for the same depth and stratification, but in its nonhydrostatic limit, according to Equation (3).

$$
\frac{d^{2} \phi}{d z^{2}}+\left[\frac{N^{2}(z)}{(U-c)^{2}}-\frac{d^{2} U / d z^{2}}{U-c}-k^{2}\right] \phi=0, \phi(0)=\phi(-H)=0
$$

Here $\Phi$ is the modal structure function, $\mathrm{k}$ is the wavenumber (also called the nonhydrostatic term), $\mathrm{U}$ is the velocity of the flow along the waves' direction of propagation (i.e. barotropic tides), and $\mathrm{H}$ is the local depth, which must be considered constant ${ }^{[28]}{ }^{[29]}$. Now, in the nonhydrostatic approximation, assuming a wavelength of $800 \mathrm{~m}$ obtained from the SAR image (see Fig. 3c), the phase speeds of the first two modes are 1.36 and $0.63 \mathrm{~m} / \mathrm{s}$, respectively. Hence, the second mode gives the most realistic phase speed when compared to the value obtained from the radar image. Consequently our hypothesis points to the existence of shorter-scale mode-2 ISWs visible in the SAR coupled with mode-4 interfacial internal tides. The phase speeds of the mode-2 ISWs and the larger-scale internal tidal waves of the fourth mode are close, permitting a resonance coupling of the two wave systems in a similar fashion reported in [30], [31] and [12]. It is possible that this resonance occurs thanks to the existence of neighboring thermoclines, as proposed for the first time in Eckart $(1961)^{[7]}$.

\section{CONCLUSION}

The present study was undertaken to detect and observe the nature of the internal tide and internal solitary waves along the Ten Degree Channel in the Andaman Sea of the Indian Ocean. In this particular stretch of the Andaman Sea, the large mode-1 solitons reported in [1] are not observed, and instead much smaller features with a very different character were detected in a number of SAR images. There, wave packets of mode-2 ISWs were unambiguously identified, and appear to be associated with a high mode interfacial internal tide. Our results indicate that the generation mechanism of these mode-2 ISWs is consistent with the beam scattering of an internal tide beam into the seasonal thermocline according to the work in [10]. The concept of a generation mechanism originally proposed by Osborne and Burch $^{[1]}$, who established that a "sufficiently localized" depression of the thermocline (a warm trough) could develop from the effects of current flow over uneven bathymetry into a train of solitary waves, needs to be revisited in the light of the new findings reported here. New remote sensing evidence of large amplitude solitons in the Andaman Sea reveals many vertical modes and complexity previously undocumented and that need to be accounted for in future research.

\section{ACKNOWLEDGEMENTS}

SAR image data were provided by the European Space Agency (ESA) under project AOPT-2423. J.M. Magalhaes is grateful for an FCT research grant (SFRH/BPD/84420/2012). 


\section{REFERENCES}

[1] Osborne, A.R. and T.L. Burch, "Internal Solitons in the Andaman Sea," Science New Series 208(4443), 451 460 (1980).

[2] Alpers, W., "Theory of radar imaging of internal waves," Nature (London) 314, 245-247 (1985).

[3] Baines, P. G., “On internal tide generation models," Deep Sea Res. Part A 29, 307- 338 (1982).

[4] Perry, B. R., G. R. Schimke, "Large-amplitude internal waves observed off the north-west coast of Sumatra," J. Geophys. Res. 70, 2319-2324 (1965).

[5] Apel, J.R, NASA Spec. Publ. SP-412 2 (1978).

[6] Korteweg, D. J., G. de Vries, "On the change of long waves advancing in a rectangular canal and a new type of long stationary waves," Phil. Mag. 5, 422 (1895).

[7] Eckart, C., "Internal Waves in the Ocean," Physics of Fluids (1958-1988) 4, 791 (1961).

[8] Gerkema T., J.T.F. Zimmerman, “An introduction to internal waves. Lecture notes," Royal NIOZ, Texel, 1-207 (2008).

[9] Shroyer E.L., J.N. Moum, J.D. Nash, "Mode 2 waves on the continental shelf: ephemeral components of the nonlinear internal wavefield," J Geophys Res. 115, C07001 (2010).

[10] Grisouard, N., C. Staquet, T. Gerkema, "Generation of internal solitary waves in a pycnocline by an internal wave beam: a numerical study,” J Fluid Mech. 676, 491-513 (2011).

[11] Mercier, M.J., M. Mathur, L. Gostiaux, T. Gerkema, J.M. Magalhaes, J.C.B. da Silva, T. Dauxois, "Soliton generation by internal tidal beams impinging on a pycnocline: laboratory experiments," J Fluid Mech. 704, 3760 (2012).

[12] da Silva, J.C.B., M.C. Bujsman, J.M. Magalhaes, "Internal waves on the upstream side of a large sill of the Mascarene Ridge: a comprehensive view of their generation mechanisms and evolution," Deep-Sea Research I 99, 87-104 (2015).

[13] Magalhaes, J.M., J. C. B. da Silva, M. C. Buijsman, C. A. E. Garcia, "Effect of the North Equatorial Counter Current on the generation and propagation of internal solitary waves off the Amazon shelf (SAR observations)," Ocean Sci. 12, 243-255 (2016).

[14] Pingree, R.D., A.L. New, "Downward propagation of internal tidal energy into the Bay of Biscay," Deep-Sea Res. Part A 36, 735-758 (1989).

[15] Pingree, R.D., A.L. New, "Abyssal penetration and bottom reflection of internal tidal energy in the Bay of Biscay,” J Phys Oceanogr. 21, 28-39 (1991).

[16] New, A.L., J.C.B. da Silva, "Remote-sensing evidence for the local generation of internal soliton packets in the central Bay of Biscay,” Deep-Sea Res Part I 49, 915-934 (2002).

[17] Azevedo, A., J.C.B. da Silva, A.L. New, "On the generation and propagation of internal waves in the southern Bay of Biscay,” Deep-Sea Res Part I 53(6), 927-941 (2006).

[18] da Silva, J.C.B., A.L. New, A. Azevedo, "On the role of SAR for observing local generation of internal solitary waves off the Iberian Peninsula," Can. J. Remote Sens. 33, 388-403 (2007).

[19] da Silva, J.C.B., A.L. New, J.M. Magalhaes, "Internal solitary waves in the Mozambique Channel: observations and interpretation," J Geophys Res. 114, C05001 (2009).

[20] Colosi, J.A., R.C. Beardsley, J.F. Lynch, G. Gawarkiewicz, C.S. Chiu, A. Scotti, "Observations of nonlinear internal waves on the outer New England continental shelf during the summer Shelf break Primer study," J Geophys Res. 106(C5), 9587-9601 (2001).

[21] Merrifield, M.A., P.E. Holloway, "Model estimates of M2 internal tide energetics at the Hawaiian Ridge," J Geophys Res.” 107(C8), 3179 (2002).

[22] Niwa, Y., T. Hibiya, "Three-dimensional numerical simulation of M2 internal tides in the East China Sea," J Geophys Res, 109 (C4), C04027 (2004). 
[23] Egbert, G.D., S.Y. Erofeeva, "Efficient inverse modeling of barotropic ocean tides," J. Atmos. Oceanic Technol. 19, 183-204 (2002).

[24] Smith, W.H.F., D.T. Sandwell, "Global sea floor topography from satellite altimetry and ship depth soundings," Science 277(5334), 1956-1962 (1997).

[25] Pingree, R.D., G.T. Mardell, A.L. New, "Propagation of internal tides from the upper slopes of the Bay of Biscay," Nature 321, 154 - 158 (1986).

[26] da Silva, J.C.B., A.L. New, J.M. Magalhaes, "On the structure and propagation of internal solitary waves generated at the Mascarene Plateau in the Indian Ocean,” Deep-Sea Res. I 58, 229-240 (2011).

[27] Buijsman, M.C., Y. Kanarska, J.C. McWilliams, "On the generation and evolution of nonlinear internal waves in the South China Sea,” J. Geophys. Res., 115, C02012 (2010).

[28] Miles, J.W., "On the stability of heterogeneous shear flows," J. Fluid Mech. 10(4), 496-508 (1961).

[29] Smyth, W. D., J.N. Moum, J.D. Nash, "Narrow band oscillations in the upper equatorial ocean, Part II: properties of shear instabilities," J. Phys. Oceanogr. 41(3), 412-428 (2011).

[30] Guo, C., V. Vlasenko, W. Alpers, N. Stashchuk, X. Chen, "Evidence of short internal waves trailing strong internal solitary waves in the northern South China Sea from synthetic aperture radar observations," Remote Sens. Environ. 124, 542-550 (2012).

[31] Vlasenko, V., N. Stashchuk, C. Guo, X. Chen, "Multimodal structure of baroclinic tides in the South China Sea," Nonlinear Process. Geophys. 17, 529-543 (2010).

[32] Gerkema, T., "Internal and interfacial tides: beam scattering and local generation of solitary waves," J. Mar. Res. 59, 227-255 (2001).

[33] Maxworthy, T., "A note on internal solitary waves produced by tidal flow over a three-dimensional ridge," J. Geophys. Res. 84(C1), 338 - 346 (1979).

[34] Chen, Z.-W., J. Xie, D. Wang, J.-M. Zhan, J. Xu, and S. Cai, "Density stratification influences on generation of different modes internal solitary waves,” J. Geophys. Res. Oceans 119, 7029-7046 (2014). 